in vivo $32: 905-910(2018)$

doi:10.21873/invivo.11327

\title{
Under-stage and Overlook of Peritoneal Spread from Bladder Urothelial Carcinoma
}

\author{
CHUANG-CHI LIAW $^{1}$, CHENG-KENG CHUANG ${ }^{2}$, YING-HSU CHANG ${ }^{2}$, \\ JOHN WEN-CHENG CHANG ${ }^{1}$, TZU-YAO LIAO ${ }^{1}$ and YU-HSIANG JUAN ${ }^{3}$ \\ ${ }^{1}$ Division of Hemato-Oncology, Department of Internal Medicine, ${ }^{2}$ Department of Urology, \\ ${ }^{3}$ Department of Medical Imaging and Intervention, Chang-Gung Memorial Hospital and \\ Chang-Gung University College of Medicine, Taoyuan, Taiwan, R.O.C.
}

\begin{abstract}
Background/Aim: Bladder cancer can spread from the sub-peritoneal space superior and posterolateral to the peritoneal cavity via the peritoneal lining. The aim of this study was to improve the identification of peritoneal spread from bladder urothelial carcinoma based on computed tomography (CT) scans. Materials and Methods: This is a retrospective study including patients selected with the following criteria: (i) pathology-confirmed urothelial carcinoma; (ii) peritoneal spread identified on CT scans from axial and corona views, either initially or after radical/partial cystectomy, concomitant chemoradiotherapy (CCRT), or radiotherapy. One hundred and fifty-nine cases met the selection criteria. Results: Routes of spread to the peritoneum included the superior to anterior direction in 59 patients $(37 \%)$, the superior to posterolateral direction in $19(12 \%)$, and the superior to both anterior and posterolateral directions in $81(51 \%)$. Invasion of specific sites included the abdominal wall in 101 patients (70\%), bowel/mesentery in 84 (53\%), prostate, uterus, and rectum in 30 (19\%), and circumferential tumors that outlined the whole bladder wall in 59 (37\%). Initial modes of therapy were chemotherapy in 86 patients (54\%), cystectomy in 55 (35\%), CCRT in eight (5\%), radiotherapy in two (1\%), and no therapy in eight (5\%). Peritoneal spread due to under-staging (clinical/pathological stage) after local therapy was found in 84 patients (53\%). Conclusion: Initial pre-therapeutic staging is easily
\end{abstract}

This article is freely accessible online.

Correspondence to: Chuang-Chi Liaw, MD, Division of HematoOncology, Department of Internal Medicine, Chang-Gung Memorial Hospital, 5 Fusing St., Gueishan Township, Taoyuan City, 333, Taiwan, R.O.C. Tel: +886 33281200 ext 8825, Fax: +886 3278211, e-mail: e102309@adm.cgmh.org.tw

Key Words: Bladder cancer, urothelial carcinoma, peritoneum, computed tomography, therapy. overlooked regarding peritoneal spread from bladder urothelial carcinoma. Combined axial and coronal views of CT scans can help identify peritoneal involvement.

The bladder is a sub-peritoneal organ, whereby the peritoneum covers the posterosuperior surface of the bladder. Along the lateral margins, the peritoneum passes onto the lateral walls of the pelvis, while the anterior, lateral, and lower parts of the posterior surface are devoid of peritoneum (1). The peritoneum is divided into two types: the visceral and parietal peritoneum. The parietal peritoneum is attached to the abdominal and the pelvic wall (1). Hence, bladder cancer can spread from the sub-peritoneal space superior and posterolateral to the peritoneal cavity via the peritoneal lining.

According to the 7 th edition of the American Joint Committee on Cancer (AJCC) TNM system, stage T4b bladder cancer describes a tumor that has spread to the pelvic or abdominal wall (2). Tumors can enter the abdominal wall from the bladder dome and the peritoneum and can invade the pelvic sidewall from the posterolateral bladder wall and/or involve the peritoneal reflection $(3,4)$. Spread of bladder urothelial carcinoma to the peritoneum is encountered (3-8). Peritoneal involvement is a metastatic disease. According to EUA (European association of Urology) guideline, local therapy such as radical cystectomy, radiation or concomitant chemoradiotherapy (CCRT) are inadequate. Cisplatin-based combination chemotherapy is standard of mode in fit patients (9).

Computed tomography (CT) scans are useful for determining the stage or extent of bladder cancer (10-12). Lee et al. reported that accurate local staging of bladder urothelial carcinoma is crucial for management (10). Raman SP et al. reported the underrated role of CT scans in bladder malignancies (11). However, identifying peritoneal spread before treatment to determine the best course of action is difficult. In this case series study, bladder urothelial carcinoma with peritoneal involvement was investigated by retrospectively reviewing CT scans. 


\section{Materials and Methods}

Study population. A retrospective case series study was conducted using data collected from patients with bladder cancer admitted to the oncology wards of Chang-Gung Memorial Hospital, Taoyuan, Taiwan, between January 2010 and December 2016. A single medical oncologist specializing in urological cancer provided most of the data, and the majority of the patients had urothelial carcinomas. They were all hospitalized patients because they needed to undergo chemotherapy. The criteria for inclusion were: (1) pathology-confirmed diagnosis of urothelial carcinoma and (2) peritoneal spread identified on CT scans from the axial and coronal views performed either initially or after radical/partial cystectomy, CCRT (concomitant chemoradiotherapy), or radiotherapy. All pretherapy CT scans for each case were discussed in our urological cancer team meetings. Chemotherapy regimens included 5fluorouracil, leucovorin, cisplatin, and gemcitabine (Gemmis; TTY, Taipei, Taiwan).

Out of the 206 hospitalized bladder cancer patients reviewed, $159(77 \%)$ met the inclusion criteria of this study because they needed to be admitted for chemotherapy. Routes of peritoneal spread, including superior to anterior, superior to posterolateral, and superior to both anterior and posterolateral, and specific site involvement, such as invasion into the abdominal wall, bowel/mesentery, prostate, uterus, and rectum, and circumferential tumors that outlined the whole bladder wall, were determined. The bladder cancer pre-therapeutic tumor extent and stage evaluation was mainly determined from CT scans. Only tumor extent and stage of two patients were determined by magnetic resonance imaging studies. The stage was evaluated according to the 7 th edition of the AJCC TNM staging system (2). Peritoneal involvement was detected either initially or after local therapy and usually was symptomatic.

Initial modes of therapy included chemotherapy only, radical/partial cystectomy, CCRT, radiotherapy only, and no therapy. Chemotherapy regimens were 5-fluorouracil, leucovorin, cisplatin, and gemcitabine (Gemmis ${ }^{\circledR}$; TTY, Taipei, Taiwan). When renal function impairment occurred (serum creatinine greater than 2 $\mathrm{mg} / \mathrm{dl}$ ), chemotherapy with continuous maintenance was administered with carboplatin replacing cisplatin. The schedule of initial CCRT combined radiotherapy with chemotherapy. Radiotherapy of the bladder and pelvic lymphatics used a 10- or 15MV X-ray dose with a parallel-opposed anteroposterior or four-field box beam. The total dose was 44-45 Gy in 22-26 fractions and further boosted to a total dose of 60-61.2 Gy.

\section{Results}

One hundred and fifty-nine consecutive bladder cancer patients, including 104 men and 55 women (range $=40-93$ years; median age: 69), met the selection criteria for this study. The patients' clinical characteristics are shown in Table I. These patients had peritoneal involvement with various tumor extents. Routes of spread to the peritoneum included the superior to anterior direction in 59 patients (37\%), superior to posterolateral in $19(12 \%)$, and superior to both anterior and posterolateral in $81(51 \%)$. For invasion of specific sites, 101 patients $(70 \%)$ had a tumor in the
Table I. Characteristics of the 159 bladder urothelial carcinoma patients with peritoneal involvement.

\begin{tabular}{lc}
\hline Characteristics & No. of patients $(\%)$ \\
\hline Age (years) & \\
$\quad$ Median & $69(40-93)$ \\
Gender & $104 / 55$ \\
$\quad$ Male/Female & \\
Routes of tumor spread - no./total no. (\%) & $59 / 159(37)$ \\
Superior to anterior & $19 / 159(12)$ \\
Superior to posterolateral & $81 / 159(51)$ \\
Superior to both anterior and posterolateral & \\
Specific site involvement - no./total no. $(\%)$ & $111 / 159(70)$ \\
Abdominal wall & $84 / 159(53)$ \\
Bowel/mesenteric & $59 / 159(37)$ \\
Prostate, uterus, and rectum & $30 / 159(19)$ \\
Entire bladder wall & \\
Initial mode of therapy - no./total no. (\%) & $86 / 159(54)$ \\
Chemotherapy & $55 / 159(35)$ \\
Radical/partial cystectomy & $8 / 159(5)$ \\
CCRT* & $2 / 159(1)$ \\
Radiotherapy & $8 / 159(5)$ \\
No treatment &
\end{tabular}

*CCRT: Concomitant chemoradiotherapy.

abdominal wall, 84 (53\%) in the bowel/mesentery, 30 (19\%) in the prostate, uterus, and rectum, and $59(37 \%)$ had a circumferential tumor that outlined the whole bladder wall.

Different types of spread were diagnosed. CT scans from axial and coronal views can help detect peritoneal spread. Bladder tumor spread in the superior to anterior direction are demonstrated in Figures 1-3. A bladder tumor with intestinal and abdominal wall invasion is shown in Figure 1. Figure 2 shows a bladder tumor with intestinal invasion and urine leakage into the abdominal cavity. A bladder tumor with mesenteric metastasis is seen in Figure 3 and bladder tumor spread in the superior to posterolateral direction in Figures 4 and 5. A bladder tumor with obvious superior and posterolateral invasion is shown in Figure 4, a bladder tumor with mesenteric metastasis in Figure 5, and a bladder tumor spread in the superior to both anterior and posterolateral directions in Figures 6 and 7. A bladder tumor with prostate invasion shown in Figure 6 reveals a circumferential bladder tumor that outlines the entire bladder wall (Figure 7).

The study included 7 patients with simultaneous bladder and renal pelvis urothelial carcinoma and 12 patients with upper urinary tract urothelial carcinoma who had previously undergone nephroureterectomy. Three cases that have had prior renal transplantation were included in this study.

Initial modes of therapy included chemotherapy in 86 patients (54\%), cystectomy in $55(35 \%)$, CCRT in eight (5\%), radiotherapy in two $(1 \%)$, and no therapy in eight $(5 \%)$. 

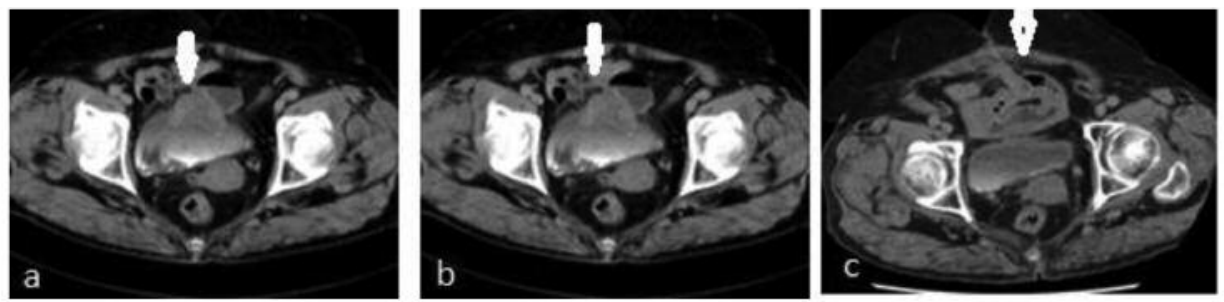

Figure 1. An 85-year-old female patient with bladder urothelial carcinoma receiving CCRT (concomitant chemoradiotherapy) showed superior to anterior extent involving the peritoneum and intestine invasion. CT scan images from (a) the coronal view and (b) axial view showed a bladder tumor with superior and anterior spread involving the peritoneum and intestine invasion. The CT scan image from (c) the axial view showed a tumor with worsening intestinal spread and abdominal wall invasions after CCRT. Arrows indicate bladder tumor with peritoneal spread.
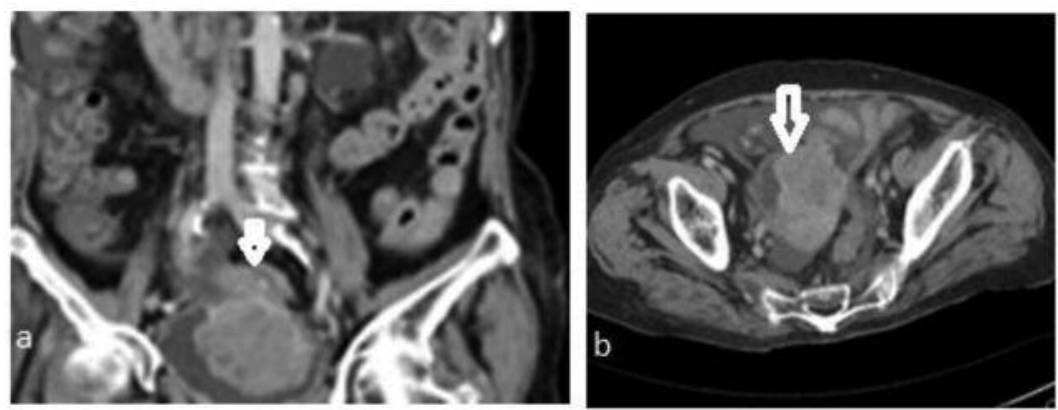

Figure 2. An 82-year-old female patient with bladder urothelial carcinoma receiving no therapy showed superior to anterior extent involving the peritoneum and urine leakage from the bladder. CT scan images from (a) the coronal view and (b) axial view showed urine leakage from the bladder with fluid accumulation. Arrows indicate bladder tumor with peritoneal spread.
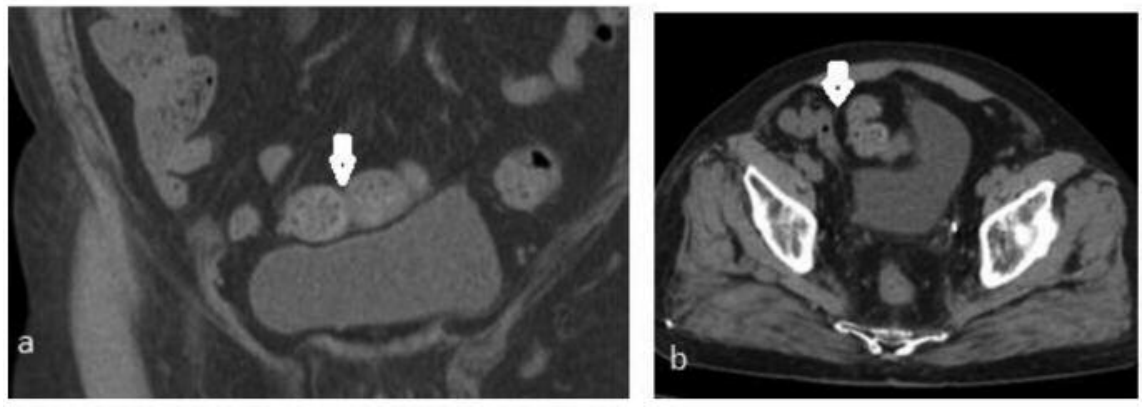

Figure 3. 71-year-old male patient with bladder urothelial carcinoma initially receiving chemotherapy showed superior to anterior extent with mesentery metastases. CT scan images from (a) the coronal view and (b) axial view showed superior and anterior tumor spread with peritoneum metastases. Arrows indicate bladder tumor with peritoneal spread.

Peritoneal spread due to under-staging (clinical/pathological stage) after radical/partial cystectomy, CCRT, or radiotherapy was found in 84 patients (53\%) despite the fact that cases had been discussed at urological cancer team meetings. Tumor staging was determined as $\mathrm{T} 1$ in five patients, $\mathrm{T} 2$ in $23, \mathrm{~T} 3$ in $26, \mathrm{~T} 4 \mathrm{a}$ in $19, \mathrm{~N} 1 \mathrm{in}$ eight, and $\mathrm{N} 2$ in three.

\section{Discussion}

Patterns of bladder urothelial carcinoma with peritoneal involvement are rarely mentioned. Lymph nodes, bones, lung, liver, and peritoneum are the most common sites of metastasis from bladder cancer $(5,6)$ and $\mathrm{T} 4 \mathrm{~b}$ bladder 

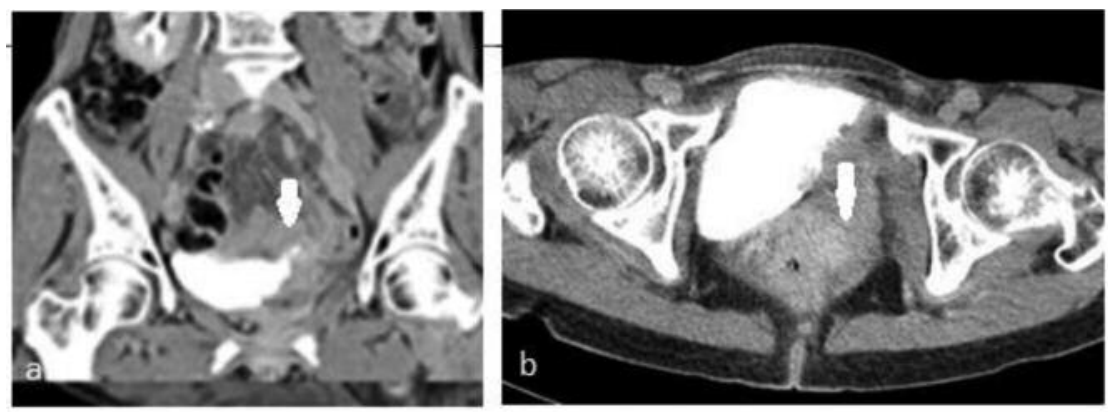

Figure 4. A 65-year-old female patient with bladder urothelial carcinoma initially receiving anterior exenteration including cystectomy showed superior to posterolateral extent with peritoneum involvement. CT scan images from (a) the coronary view and (b) axial view showed superior and posterolateral tumor spread.
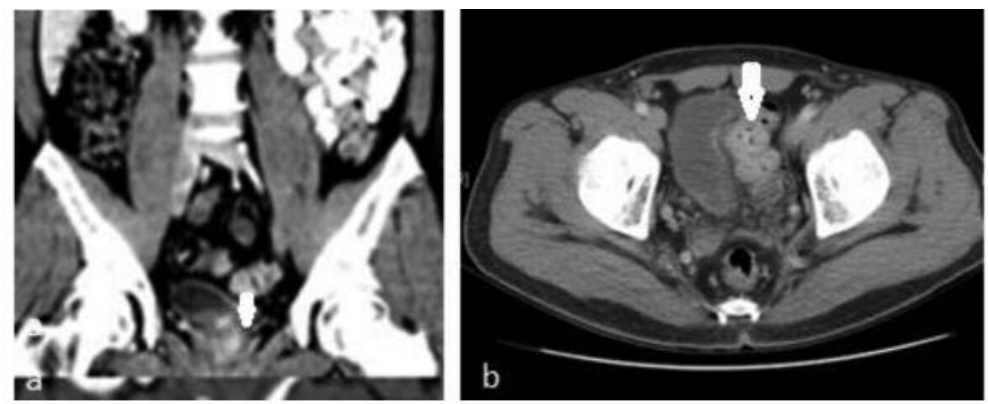

Figure 5. A 54-year-old male patient with bladder urothelial carcinoma initially receiving chemotherapy showed superior to posterolateral extent with mesenteric metastases. CT scan images from (a) the coronal view and $(b)$ axial view showed posterolateral tumor spread with mesenteric metastases. Arrows indicate bladder tumor with peritoneal spread.
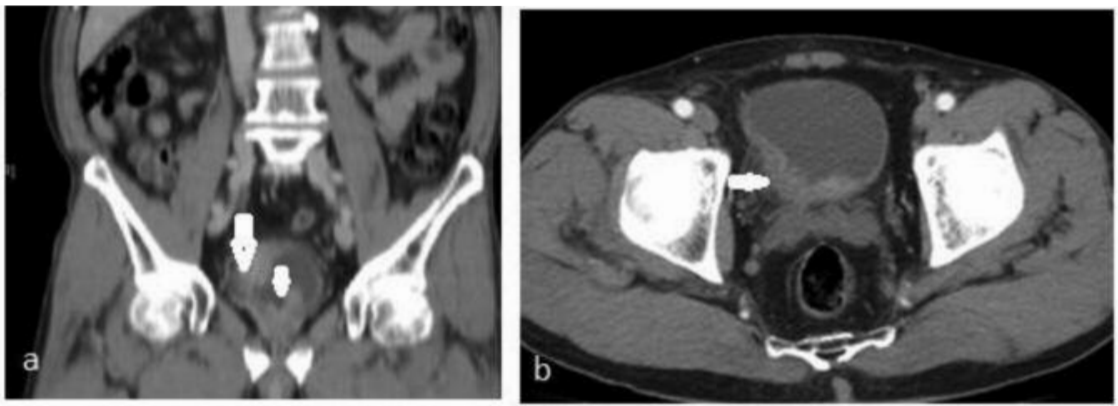

Figure 6. A 66-year-old male patient with bladder urothelial carcinoma initially receiving radical cysto-prostatectomy showed superior to both anterior and posterolateral extents involving the peritoneum and prostate invasion. CT scan images from (a) the coronal view and (b) axial view showed anterior, superior, and posterior tumor spread involving the peritoneum with abdominal wall and prostate invasion. Arrows indicate bladder tumor with peritoneal spread.

cancers are likely to already involve the peritoneum (2-4). However, identifying peritoneal spread before treatment to determine the best course of action is difficult because of a dirty and/or thickened peritoneum don't represent tumor involvement. Black et al. reported that long-term survival can be achieved in some patients with cT4b bladder cancer undergoing chemotherapy and extirpative surgery (13). However, Liberman et al. mentioned that radical cystectomy of pT4b patients had higher cancer-specific mortality (14). 


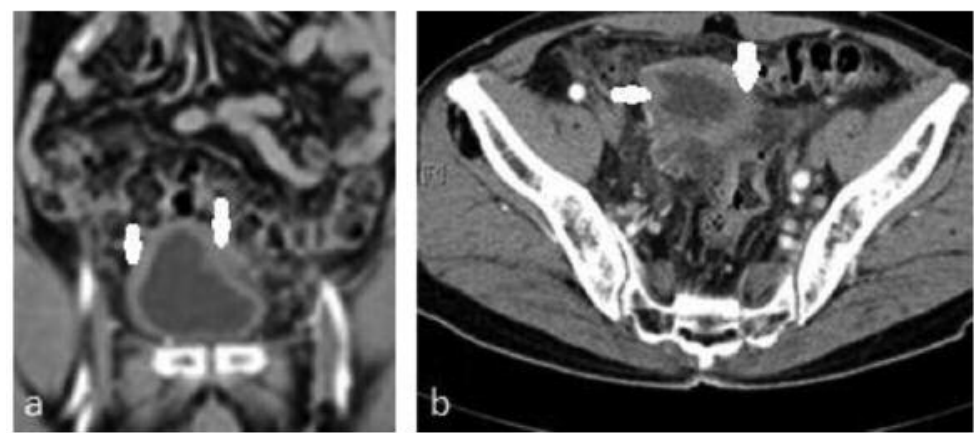

Figure 7. An 80-year-old male patient with bladder urothelial carcinoma initially receiving CCRT (concomitant chemoradiotherapy) showed superior to both anterior and posterolateral extents involving the peritoneum that were circumferential around the whole bladder wall. CT scan images from (a) the coronal view and (b) axial view showed superior tumor spread around the entire upper portion of bladder wall. Arrows indicate bladder tumor with peritoneal spread.

CT scans have a role in the local staging of urothelial carcinoma $(10,11)$ and are important in the diagnosis of peritoneal spread (7-9). Hence, understanding the anatomy of the peritoneum and bladder is crucial (1). Based on our study, bladder tumors can spread from the bladder dome in a superior to anterior and/or posterolateral direction. Tumors that spread from the superior and anterior extent can invade the intestine/mesentery and abdominal wall, while tumors that spread posterolateral can invade the prostate, uterus, or rectum. Tumors can also be circumferential and outline the whole bladder wall. CT scans from axial and coronal views helped in the detection of peritoneal spread in our cases.

CT scans can influence the initial therapy modality. More than half of our patients had under-staging and underwent local therapy with radical/partial cystectomy, CCRT, or radiotherapy. Patients with clinical under-staging experienced incomplete removal of the bladder tumor or had peritoneal lesions outside the radiation field. These tumors usually progress after local therapy. This is inconsistent with the results of our study, which suggested that CCRT is a feasible and promising treatment for invasive bladder cancer (15). However, recently published articles show that these patients benefit from continuous maintenance of cisplatin-based chemotherapy.

Despite the fact that cases are discussed at urological cancer team meetings, incorrect clinical staging, especially under-staging, is a serious problem in bladder cancers patients, and improvements of the staging process are needed to achieve more accuracy $(16,17)$. Pathological understaging of patients is often seen because the cancer is mistaken as T3b (macroscopic extravesicular spread) or T4a (prostate, uterus, or rectum involvement). CT scans with combined axial and coronal views can reduce clinical understaging, especially regarding peritoneal involvement.

The tumor microenvironment has been shown to impact tumor progression, and key factors have been detected in bladder cancer tissues (18). Evidence suggests that the critical factors of the tumor microenvironment are immune and inflammatory cells, blood and lymphatic vascular networks, fibroblasts, and the extracellular matrix (18). Cancer-associated fibroblasts (CAFs), a major component of the cancer stroma, have a prominent role in the progression, growth, and spread of cancers $(19,20)$, and can be involved in angiogenesis and the response to injury. A dirty and thickened peritoneum represented cancer cells in the peritoneum; however, the burden of cancer cells and their fibrotic tissue could not be determined.

Our study has several important limitations. First, the data were collected mainly from one physician as a retrospective case cohort study in a single center. Second, the diagnosis of peritoneal involvement was not verified by tissue confirmation.

\section{Conclusion}

Initial pre-therapeutic staging is easy to overlook for peritoneal spread from bladder urothelial carcinoma. Combined axial and coronal views of CT scans can help identify peritoneal involvement and avoid clinical understaging of bladder cancer.

\section{Conflicts of Interest}

The Authors declare no potential conflicts of interest.

\section{Acknowledgments}

The Authors wish to acknowledge the oncology nurse staff for providing the best care for these patients.

\section{References}

1 Pannu HK and Oliphant M: The subperitoneal space and peritoneal cavity: basic concepts Abdom Imaging 40: 27102722, 2015. 
2 Edge SB, Byrd DR, Compton CC, Fritz AG, Greene FL and Trotti A: Urinary bladder. In: AJCC cancer staging manual. (7th edition) Springer, New York, NY, pp. 497-505, 2010.

3 Le O: Patterns of peritoneal spread of tumor in the abdomen and pelvis World J Radiol 5: 106-112, 2013.

4 Griffin N, Burke C and Grant LA: Common primary tumors of the abdomen and pelvis and their patterns of tumor spread as seen on multi-detector computed tomography. Insights Imaging 2: 205-214, 2011.

5 Shinagare AB, Ramaiya NH, Jagannathan JP, Fennessy FM, Taplin ME and Van den Abbeele AD: Metastatic pattern of bladder cancer: correlation with the characteristics of the primary tumor. AJR Am J Roentgenol 196: 117-122, 2011.

6 Turkbey B, Basaran C, Karcaaltincaba M, Akpinar E, Oguz B, Akata D, Ozmen MN and Akhan O: Peritoneal carcinomatosis in urinary bladder cancer. Clin Imaging 32: 192-195, 2008.

7 Chrysikopoulos H, Maniatis V, Roussakis A, Pappas J and Andreou J: Peritoneal metastases from transitional cell carcinoma of the urinary tract: CT and MR imaging. Abdom Imaging 23: 91 95, 1998.

8 Pevarski DJ, Mergo PJ and Ros PR: Peritoneal carcinomatosis due to transitional cell carcinoma of the bladder: CT findings in two patients. AJR Am J Roentgenol 164: 929-930, 1995.

9 Milowsky MI, Rumble RB, Booth CM, Gilligan T, Eapen LJ, Hauke RJ, Boumansour P and Lee CT: Guideline on muscleinvasive and metastatic bladder cancer (European Association of Urology Guideline): American Society of Clinical Oncology Clinical Practice Guideline Endorsement. J Clin Oncol 34: 19451952, 2016.

10 Lee $\mathrm{CH}$, Tan $\mathrm{CH}$, Faria SC and Kundra V: Role of imaging in the local staging of urothelial carcinoma of the bladder. AJR Am J Roentgenol 208: 1193-1205, 2017.

11 Raman SP and Fishman EK: Bladder malignancies on CT: the underrated role of CT in diagnosis. AJR Am J Roentgenol 203: 347-354, 2014.

12 Milowsky MI, Rumble RB, Booth CM, Gilligan T, Eapen LJ, Hauke RJ, Boumansour P and Lee CT: Guideline on muscleinvasive and metastatic bladder cancer (European Association of Urology Guideline): American Society of Clinical Oncology Clinical Practice Guideline Endorsement. J Clin Oncol 34: 1945$1952,2016$.
13 Black PC, Dinney CP, Brown GA, Kassouf W, Siefker-Radtke AO, Munsell MF, Grossman HB and Kamat AM: The role of radical cystectomy in patients with clinical T4b bladder cancer. Urol Oncol 29: 157-161, 2011.

14 Liberman D, Alasker A, Sun M, Ismail S, Lughezzani G, Jeldres C, Budaus L, Thuret R, Shariat SF, Widmer H, Perrotte P, Graefen M, Montorsi F and Karakiewicz PI: Radical cystectomy for patients with pT4 urothelial carcinoma in a large populationbased study. BJU Int 107: 905-911, 2011.

15 Chen WC, Liaw CC, Chuang CK, Chen MF, Chen CS, Lin PY, Chang PL, Chu SH, Wu CT and Hong JH: Concurrent cisplatin, 5-fluorouracil, leucovorin, and radiotherapy for invasive bladder cancer Int J Radiat Oncol Biol Phys 56: 726-733, 2003.

16 Liaw CC, Chuang CK, Chang YH, Liao TY, Chang JW and Juan YH: Bladder urothelial carcinoma with peritoneal involvement: benefit of continuous maintenance chemotherapy. Anticancer Res 37: 6443-6451, 2017.

17 Turker P, Bostrom PJ, Wroclawski ML, van Rhijn B, Kortekangas H, Kuk C, Mirtti T, Fleshner NE, Jewett MA, Finelli A, Kwast TV, Evans A, Sweet J, Laato M and Zlotta AR: Upstaging of urothelial cancer at the time of radical cystectomy: factors associated with upstaging and its effect on outcome. BJU Int 110: 804-811, 2012.

18 Wang M, Zhao J, Zhang L, Wei F, Lian Y, Wu Y, Gong Z, Zhang S, Zhou J, Cao K, Li X, Xiong W, Li G, Zeng Z and Guo C: Role of tumor microenvironment in tumorigenesis. J Cancer 8 : 761-773, 2017.

19 Kalluri R: The biology and function of fibroblasts in cancer. Nat Rev Cancer 16: 582-598, 2016.

20 Midwood KS, Williams LV and Schwarzbauer JE: Tissue repair and the dynamics of the extracellular matrix. Int J Biochem Cell Biol 36: 1031-1037, 2004.

Received March 31, 2018

Revised April 24, 2018

Accepted April 25, 2018 